\title{
A Simple Image-Adaptive Watermarking Algorithm with Blind Extraction
}

\author{
Mohamed Hamid and Chunyan Wang \\ Department of Electrical and Computer engineering, Concordia University, Montreal, Canada \\ 1455 de Maisonneuve West, Montreal, Canada H3G1M8 \\ $\{$ mo_hami,chunyan\}@ece.concordia.ca
}

\begin{abstract}
In this paper, we propose an adaptive, DCT based, blind image watermarking algorithm. The adaptability is developed, while exploring the properties of the human visual system (HVS), to embed robust watermarks in DCT matrices while making them the least visible in the space domain. In the algorithm, binary watermarks are embedded in image blocks having gray level variations sufficient to mask a certain level of signal alterations. The orientations of the variations in such a block are detected by examining the locations of the most significant DCT coefficients in the matrix and a particular DCT coefficient is then chosen for watermark embedding with a view to making the gray level changes resulting from the embedding have a similar orientation with the original image signal to mask the watermark better. Moreover, the embedding strength is made to depend on the DC component, as it indicates the brightness of the image block, to further reduce the visibility of the watermark. The algorithm is computationally simple because it extracts the needed features directly from the DCT coefficients. Simulation results show that the algorithm is highly robust against JPEG compression even at low quality factors while maintaining high visual quality. Furthermore, the computational simplicity of the algorithm makes it very useful in real-time applications.
\end{abstract}

Keywords - Digital image watermarking, adaptive embedding, blind extraction, DCT, Human Visual System, JPEG compression.

\section{INTRODUCTION}

Digital image watermarking has emerged as an important technique for copyright protection and image authentication among other applications. As data transformation processes such as JPEG compression are widely used for image storage and transmission, embedded watermarks need to be robust enough to survive such kinds of transformations. Moreover, it is often required that the watermarks are made imperceptible to preserve the visual quality of the original images. As the robustness and the imperceptibility usually conflict each other, one would try to find a good trade-off between them. The work presented in this paper targets a challenging issue of embedding robust watermarks while maintaining their imperceptibility.

Watermarks embedded in a transform domain are generally considered more robust than those in spatial domain [1]. As image data quantization and compression are often performed in the DCT domain, DCT watermarking is very common in practice. In order to improve the robustness, a scheme to embed the watermark in the perceptually most significant components was proposed in [1], whereas Lee and Li chose to embed their watermarks in the lowest-frequency DCT coefficients [2].
To tackle the robustness-imperceptibility conflict, some DCT-based schemes involving the use of the human visual system (HVS) properties have been reported. According to the visual model developed by Watson for adaptive image quantization, the HVS is normally less sensitive to image data alterations in brighter regions or regions with higher background textures [3]. Podilchuk and Zeng used Watson's model to determine the upper bounds on watermark insertion in the DCT domain [4]. In the work reported in [5] the just noticeable distortion (JND) models in the DCT domain were used and their effectiveness was assessed. Embedding techniques may differ by watermark strength versus frequency ranges and/or texture levels. Some added the watermark to image singularities in the high frequency bands in the DWT domain [6] or only to image blocks of strong texture [7] to preserve the visual quality, whereas others may choose the DC coefficient for watermark embedding to improve the robustness $[8,9]$. The method reported in [10] is to embed the watermarks in one of the three lowest AC frequency components according to the texture directions.

In this paper, we propose an adaptive DCT-based watermarking algorithm that embeds the watermark in blocks that contain sufficient gray level variations and chooses the location of watermark embedding according to the direction of those variations. In this way, while preserving good visual quality, the embedded watermark can be made robust to data processes such as JPEG compression. The algorithm uses a binary watermark, which can be extracted blindly from watermarked images. The embedding and the extraction processes are very simple for an easy implementation of the algorithm.

\section{PRINCIPLE OF THE ALGORITHM}

As mentioned previously, the work presented in this paper aims at developing a simple algorithm for embedding, in the DCT domain, an invisible binary watermark that is robust against image data processes such as quantization or compression. To achieve this objective, the work is focused on the following issues for an image-adaptive watermarking.

- Defining the image features that can be used to mask the data alteration resulting from watermark embedding;

- Determining an effective but simple method to detect the features;

- Incorporating the information of the detected image features in the two elements in the embedding process, i.e., 
the embedding strength and the frequency components in which the watermark data are embedded.

It is known that an image pattern can be better masked if the background luminance is increased and/or it appears together with other patterns, particularly if these patterns are of similar spatial frequencies and orientations [3]. In other words, an image data alteration can be made less perceptible in an area having a higher gray level mean and/or more variations. Hence, the local gray level mean and variations are the elements to be used to determine the watermarking strength to make a robust watermark imperceptible.

The gray level mean value is indicated by the DC component in the DCT coefficient matrix whereas the gray level variations in an image can be very complex in terms of amplitudes and directions in the 2-D space. However, when the image is decomposed into blocks of $8 \times 8$ pixels for data processing, the image pattern in each block becomes very simple. In most cases, the profile of the gray level variations in such a block is a simple step/ramp or ridge/valley placed in a horizontal, vertical, or diagonal direction. Four examples of such variations are illustrated in Fig. 1 (a1) (a4) and their respective DCT coefficient matrices shown in Fig. 1 (b1) (b4).

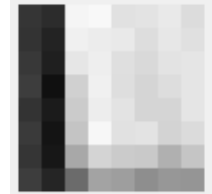

(a1)

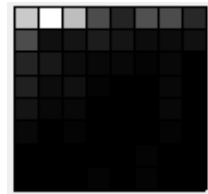

(b1)

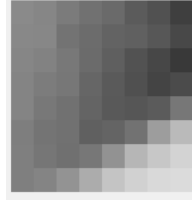

(a2)

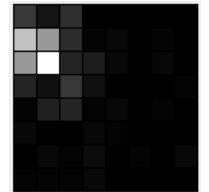

(b2)

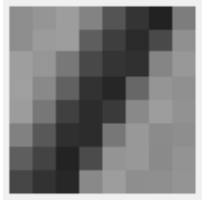

(a3)

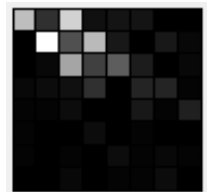

(b3)

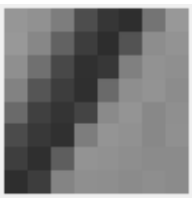

(a4)

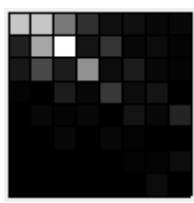

(b4)
Figure 1. (a1) (a4) Examples of gray level variation patterns in $8 \times 8$ blocks. (b1) (b4) DCT matrices corresponding to the patterns.

From the examples illustrated in Fig. 1 and other possible patterns in blocks of $8 \times 8$ pixels, one can observe that the locations of the most significant frequency coefficients, referred to as DCT peaks, in the DCT matrix indicate the direction of the gray level variation in an image block. If the watermark data is embedded in the frequency components aligned with the peaks in the original DCT matrix, the gray level variation resulting from the watermark embedding will have a similar direction to that of the original image block and it will likely be masked. In this case, the embedding strength can be increased to improve robustness without compromising the imperceptibility.

In a block of $8 \times 8$ pixels, if the gray level variations are more complex than those shown in Fig. 1, the presence of such higher-spatial-frequency patterns can make a data alteration less perceptible. Thus, it will be an "easy" case to embed imperceptible watermarks. On the contrary, in a "flat" block where there is no significant gray level variation, it will be difficult to embed invisible watermarks. Hence one may have to avoid, if possible, any significant data alteration in the blocks located in very flat regions in the absence of edges. Fortunately, in a normal case, there are sufficient "edge" blocks in an image for invisible watermarking.

Summarizing the points described above, the proposed adaptive watermarking algorithm will feature the following points.

- The watermark embedding is performed in blocks with sufficient gray level variations, i.e. at presence of significant low frequency components in its DCT matrix.

- In such a DCT matrix, the DCT peaks are localized to identify the direction of the gray level variations.

- The DCT coefficients in which watermark data is to be embedded are chosen under two conditions. Firstly, the gray level alteration caused by watermarking should be in the same direction as those in the original image block. Secondly, their frequencies are, in principle, next (or close) to those of the peaks in order to use a good energy level for watermarking without altering the main frequency components.

- The strength of watermark embedding, i.e. the quantity of the data alteration in the chosen DCT coefficient is related to the local gray level mean, i.e. the DC coefficient in the DCT matrix.

Having the above-listed features, the algorithm will embed each bit of the watermark adaptively to the local gray level variations without adding a significant amount of computation on top of that for image processing operations. In the following section, an implementation of the algorithm is presented. It should be noted that the principle presented in this section can also be applied in different ways for watermarking with some different implementation details.

\section{IMPLEMENTATION OF THE ALGORITHM}

The algorithm can be implemented by performing the following steps in each $8 \times 8$ block.

1. Calculate the DCT coefficients of the block.

2. Detect the flatness of the block. If there are enough gray level variations, continue to the next steps. Otherwise, leave it unchanged.

3. Detect the directions of the variations and choose the DCT coefficient for embedding accordingly.

4. Embed 1-bit of the watermark in the block.

5. Perform the IDCT to get the watermarked image block.

The operations required for these steps are described in the following subsections.

\section{A. Detecting the Flatness of the Block}

To preserve visual quality, data alterations in flat blocks must be avoided as discussed in Section II. Non-flat blocks can be indicated by the average of the absolute values of the low frequency DCT coefficients. If the average is higher than a given threshold $(T H)$, the block has enough variations and watermark embedding will be performed. Otherwise, the block is flat and should remain unchanged. 


\section{B. Detecting the Directions of Gray Level Variations}

The direction of gray level variations can be represented by an angle $\theta$ such that $|\theta| \leq 180^{\circ}$. In this work, the directions of variation are classified to the five directions shown in Table 1.

These directions are indicated by the locations of the two most significant DCT coefficients, referred to as the two peaks. The detection conditions, shown in Table 1, are evaluated sequentially, starting from the top. For example, if the highest peak is located in the diagonal line, the first two conditions will be checked to be false and the third one will be found true, thus, the direction will be detected as diagonal.

TABLE 1. DIRECTIONS OF GRAY LEVEL VARIATIONS (GLV) AND THEIR INDICATIONS BY THE LOCATIONS OF THE TWO PEAKS.

\begin{tabular}{|c|l|l|}
\hline GLV Directions & Example & \multicolumn{1}{c|}{ Detection Conditions } \\
\hline Horizontal & Fig. 1 (a1) & Both peaks are in the first row. \\
\hline Vertical & - & Both peaks are in the first column. \\
\hline Diagonal & Fig. 1 (a3) & $\begin{array}{l}\text { The highest peak is on the diagonal } \\
\text { line. }\end{array}$ \\
\hline Diagonal-horizontal & Fig. 1 (a4) & $\begin{array}{l}\text { The highest peak is above the diagonal } \\
\text { line. }\end{array}$ \\
\hline Diagonal-vertical & Fig. 1 (a2) & $\begin{array}{l}\text { The highest peak is below the diagonal } \\
\text { line. }\end{array}$ \\
\hline
\end{tabular}

\section{Choosing the DCT Coefficient for Embedding}

We must choose a DCT coefficient that is aligned with the peak and represents the same direction of gray level change as that in the original block. In this way, the changes caused by watermark embedding in this coefficient will be masked by the original variations and therefore less perceptible. Moreover, we will choose that coefficient to be in the middle frequency range and avoid modifying the most significant frequency coefficient.

The DCT coefficients are divided into five groups, each of which corresponds to one of the five directions illustrated in Table 1. After the direction is detected, one coefficient from its group is chosen for embedding the watermark bit.

Fig. 2 illustrates the groups and the chosen coefficient in each group. Normally we choose the coefficient that is indicated by the solid circle. However, if that coefficient happens to be the most significant in the group, we choose the coefficient indicated by the dashed circle. For example, if the gray level variations were detected to be in the vertical direction, we would choose the coefficient no. 10 in case that it is not the highest of the vertical group; otherwise we will choose no. 9.

\section{Embedding the watermark}

After choosing the DCT coefficient, the average of the absolute values of its group, denoted as $A V G$, is calculated, excluding the chosen coefficient and the highest value of the group. The coefficient is modified as shown by the following pseudocode where $C$ is the absolute value of the chosen coefficient and $w$ is the watermark bit.

\begin{tabular}{|cc|}
\hline If $w==0$ & Else \\
If $C>(A V G-K)$ & If $\mathrm{C}<(A V G+K)$ \\
$\mathrm{C}=\mathrm{AVG}-\mathrm{K} ;$ & $\mathrm{C}=\mathrm{AVG}+\mathrm{K} ;$ \\
End & End \\
& End \\
\hline
\end{tabular}

To maximize the strength of embedding without causing visible distortions, the value of $K$ is made adaptive to the gray level mean of the block, which is reflected by the DC coefficient. The value of $K$ is calculated as:

$$
K=K^{\prime}+\alpha|D C|
$$

where $K^{\prime}$ is a predetermined constant, $\alpha$ is a scaling factor and $D C$ is the value of the DC coefficient.

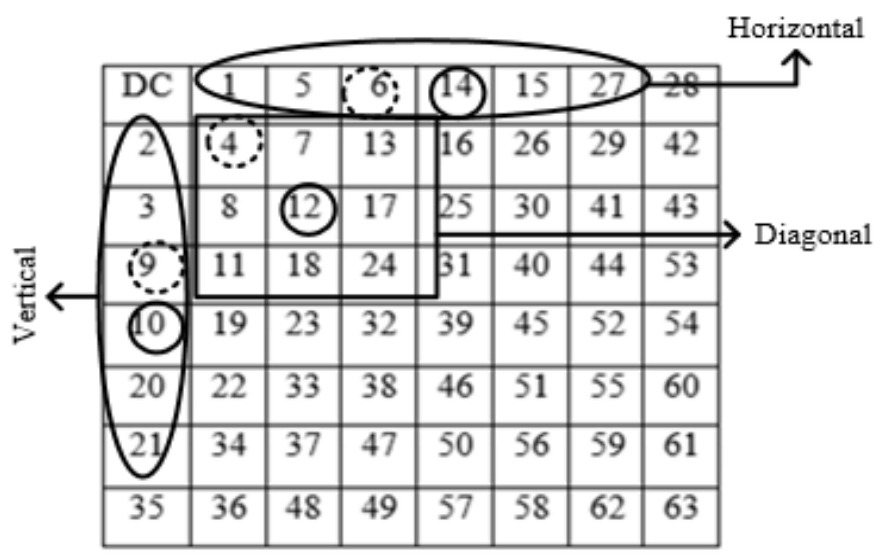

(a)

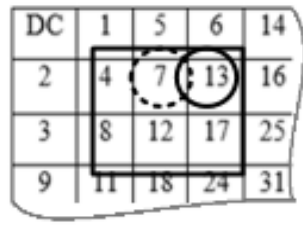

(b)

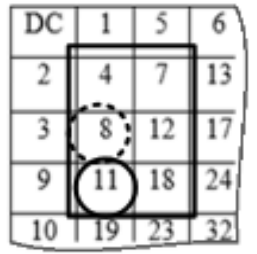

(c)
Figure 2. DCT matrix in zigzag order with the coefficient groups corresponding to the GLV directions shown in Table 1. (a) Vertical, horizontal and diagonal;

(b) Diagonal-Horizontal; and

(c) Diagonal-Vertical.

\section{E. Extracting the Watermark}

The watermark can be extracted, without the presence of the original image, from the watermarked image. It is performed in the steps described in the subsections $\mathrm{A}, \mathrm{B}$ and $\mathrm{C}$ followed by the simple operation as presented in the pseudo code:

If $\mathrm{C}>\mathrm{AVG}$
Else $\quad \mathrm{W}=1 ;$
End $\quad \mathrm{W}=0 ;$




\section{SiMULATION RESULTS}

The effectiveness of the algorithm has been evaluated using different gray scale images of size 512 x 512. A 32 x 32 binary watermark was used. The parameters $T H, K^{\prime}$ and $\alpha$ were set to be 30,10 , and 0.05 , respectively.

Fig. 3 shows an example of an original image and its watermarked version. The peak signal to noise ratio (PSNR) and the correctness rate $(\mathrm{CR})$ were used to evaluate the imperceptibility and robustness against JPEG compression, respectively. The results of the proposed algorithm were compared to those reported by You [6], Lee [2] and Zhi [5].

Table 2 shows a comparison of the CR values after JPEG compression with different quality factors. The proposed algorithm maintains consistently a CR value above $90 \%$ for the entire range, while still being able to yield $\mathrm{CR}=79.3 \%$ at $\mathrm{Q}=$ 10. In almost all the cases, it results in noticeably higher CR values than those compared do and more remarkably at low $\mathrm{Q}$ values.

Table 3 shows a comparison of the PSNR values of the watermarked images. The PSNR values of the proposed algorithm are significantly higher than the other two for all the test images listed in Table 3.

TABLE 2. COMPARISON OF CR VALUES AFTER JPEG COMPRESSION

\begin{tabular}{|c|c|c|c|c|c|}
\hline \multirow{2}{*}{ Q } & \multicolumn{5}{|c|}{ Correctness Rate (\%) } \\
\cline { 2 - 6 } & You [6] & Lee [2] & Zhi 1 [5]* & Zhi 2 [5]* & Proposed \\
\hline 100 & 100 & 100 & 100 & 100 & 100 \\
\hline 90 & N/A & N/A & 99.00 & 100 & 99.61 \\
\hline 80 & 97.35 & 97.41 & 95.00 & 98.00 & 98.93 \\
\hline 70 & N/A & N/A & 72.00 & 85.00 & 98.14 \\
\hline 60 & 95.48 & 95.94 & 72.00 & 80.00 & 98.05 \\
\hline 50 & 95.01 & 97.71 & 70.00 & 74.00 & 96.88 \\
\hline 40 & N/A & N/A & 30.00 & 70.00 & 96.09 \\
\hline 30 & N/A & N/A & 45.00 & 38.00 & 93.36 \\
\hline
\end{tabular}

$*$ Zhi 1 and Zhi 2 are based on the JND models developed by Wei and Bae respectively [5].

TABLE 3. COMPARISON OF PSNR VALUES

\begin{tabular}{|c|c|c|c|}
\hline \multirow{2}{*}{ Host Image } & \multicolumn{3}{|c|}{ PSNR (dB) } \\
\cline { 2 - 4 } & You [6] & Lee [2] & Proposed \\
\hline Lena & 40.58 & 41.29 & 43.10 \\
\hline Boat & 39.75 & 40.54 & 41.57 \\
\hline Baboon & 38.41 & 39.79 & 42.30 \\
\hline Peppers & 40.37 & 41.91 & 43.84 \\
\hline
\end{tabular}

* PSNR values for reference [5] are unavailable.

Overall, the proposed algorithm is highly robust against JPEG compression even at low quality factors while it maintains high visual quality in the watermarked image.

\section{CONCLUSION}

In this paper, an adaptive image watermarking algorithm in the DCT domain that allows for blind extraction has been proposed and its effectiveness evaluated. The algorithm embeds the binary watermark in blocks in a way that it is adaptive to the directions of the gray level variations and to the brightness of the block. It is done by embedding the watermark in the location chosen in the DCT matrix according to the locations of the most significant DCT coefficients of the original image signal and by adjusting the embedding strength according to the DC coefficient. The algorithm is computationally simple because it extracts the needed gray level characteristics directly from the DCT coefficients without extra processes. Simulation results have shown that the algorithm provides high visual quality and excellent robustness against lossy JPEG compression even at low quality factors. In addition to the high performance in these usually conflicting aspects, the computational simplicity of the algorithm makes it useful in real-time applications.

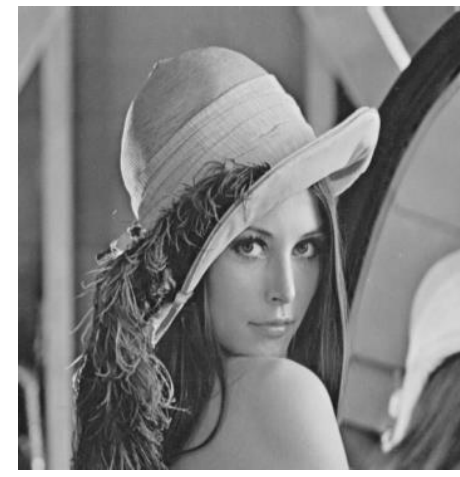

(a)

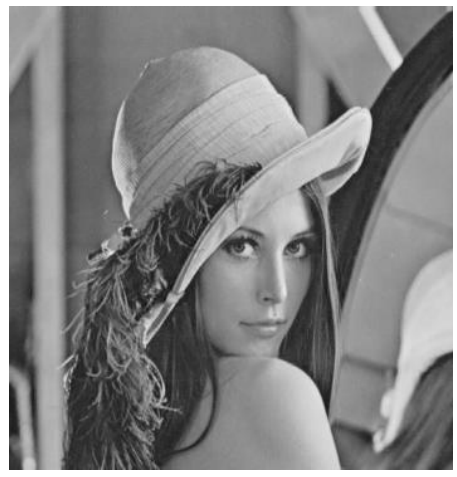

(b)
Figure 3. (a) Original image and (b) Watermarked image.

\section{REFERENCES}

[1] I.J. Cox, J. Kilian, F.T. Leighton and T. Shamoon, "Secure spread spectrum watermarking for multimedia," IEEE Transactions on Image Processing, vol. 6, pp. 1673-1687, 1997.

[2] Jung-San Lee and Bo Li, "Self-recognized image protection technique that resists large-scale cropping," IEEE MultiMedia, vol. 21, pp. 60-73, 2014.

[3] A.B. Watson, "DCT quantization matrices visually optimized for individual images," in IS\&T/SPIE's Symposium on Electronic Imaging: Science and Technology, pp. 202-216, Jan. 1993.

[4] C.I. Podilchuk and Wenjun Zeng, "Image-adaptive watermarking using visual models," IEEE Journal on Selected Areas in Communications, vol. 16, pp. 525-539, 1998.

[5] Shuang Zhi, Yana Zhang, Cheng Yang and Jianbo Liu, "Edge detection based JND model for digital watermarking," in Proc. Int. Conf. Signal Process. (ICSP), pp. 875-879, Oct. 2014.

[6] Xinge You, Liang Du, Yiu-ming Cheung and Qiuhui Chen, "A blind watermarking scheme using new nontensor product wavelet filter banks," IEEE Trans. Image Process., vol. 19, pp. 3271-3284, 2010.

[7] M. Cedillo-Hernandez, M. Nakano-Miyatake and H. Perez-Meana, "Robust watermarking to geometric distortion based on image normalization and texture classification," in 51st Midwest Symposium on Circuits and Systems (MWSCAS), pp. 245-248, 2008.

[8] Jiwu Huang, Y.Q. Shi and Yi Shi, "Embedding image watermarks in dc components," IEEE Transactions on Circuits and Systems for Video Technology, vol. 10, pp. 974-979, 2000.

[9] Xiuli Wang, Weihua Xie and Xuan Wang, "Adaptive watermarking algorithm of image based on the human visual system," in Second International Conference on Business Computing and Global Informatization (BCGIN), 2012, pp. 511-514, 2012.

[10] OuJun Lou, Li Shaohua, Liu ZhaoXia and Tang ShuangTong, "A novel multi-bit watermarking algorithm based on HVS," in Sixth International Symposium on Parallel Architectures, Algorithms and Programming (PAAP), pp. 278-281, 2014. 